УДК 622.279

\title{
МОДЕЛИРОВАНИЕ ТЕЧЕНИЯ ГАЗОЖИДКОСТНОГО ПОТОКА НА ПРИЕМЕ ЭЛЕКТРОЦЕНТРОБЕЖНЫХ НАСОСНЫХ УСТАНОВОК С КАРКАСНО-ПРОВОЛОЧНЫМ ФИЛЬТРОМ
}

\author{
Уразаков Камил Рахматуллович1, \\ urazakk@mail.ru
}

\author{
Тугунов Павел Михайлович1, \\ PavelTugunov@gmail.com
}

\author{
Алиметов Шамиль Агаметович², \\ shamil_alimetov@mail.ru \\ 1 Уфимский государственный нефтяной технической университет, \\ Россия, 450064, г. Уфа, ул. Космонавтов, 1. \\ 2 «Когалымнефтегаз», Общество с ограниченной ответственностью «ЛУКОЙЛ - Западная Сибирь», \\ Россия, г. Когалым, ул. Прибалтийская, 20.
}

\begin{abstract}
Актуальность. На сегодняшний день одним из наиболее неблагоприятных фракторов, осложняющих механизированную добычу нефти, является взаимодействие механических примесей, переносимых скважинной продукцией, с узлами внутрискважинного оборудования. Согласно статистическим исследованиям, механические примеси являются определяющей причиной возникновения осложнений неисправностей в работе установок электроцентробежных насосов.

Объект: каркасно-проволочные фрильтры, обладающие наилучшими гидравлическими характеристиками в сравнении с другими видами механических фильтров. Несмотря на широкую распространенность, на сегодняшний день не существует общепринятой методики расчета технологического режима скважин, оборудованных электроцентробежными насосами с механическими фрильтрами. Вместе с тем в работе показано, что механические фильтры за счет гидравлического сопротивления оказывают существенное влияние на геометрию линий тока жидкости в области приема насоса, следовательно, газовой фразы и фрракции механических примесей, движущихся в потоке.

Цель: исследование влияния гидравлических характеристик механических фильтров на особенности течения откачиваемой продукции в интервале приема электроцентробежного насоса; разработка математической модели многокомпонентного течения газожидкостного потока, содержащего механические примеси, в интервале приема электроцентробежной насосной установки, оборудованной каркасно-проволочным фильтром.

Результаты. Показано, что для насоса с фильтром за счет создаваемого им гидравлического сопротивления интервал приема работает более равномерно, причем по мере снижения гидравлического параметра достигается более равномерный профиль радиального притока жидкости в области приема насоса. Исследованы особенности течения пузырьков газа и взвешенных частиц механических примесей, движущихся в потоке жидкости в области приема насоса. Показано, что условия сепарации газа на приеме, а также распределение концентрации механических примесей изменяются при варьировании гидравлических характеристик фильтра в составе насоса, что необходимо учитьвать при проектировании технологического режима эксплуатации скважин. Исследовано влияние гидравлических характеристик фильтра на величину потерь давления при течении жидкости через фильтрующий элемент.
\end{abstract}

\section{Ключевые слова:}

Установка электроцентробежного насоса, каркасно-проволочный фрильтр, моделирование течения, скорость потока, механические примеси, пузырьки газа, уравнения Навье-Стокса.

\section{Введение}

В настоящее время значительная доля месторождений Российской Федерации находится на поздней стадии разработки, причем добыча нефти ведется преимущественно механизированным способом. Распределение механизированного фонда добывающих скважин по способам добычи выглядит следующим образом: самую большую часть занимают установки электроцентробежных и скважинных штанговых насосов (УЭЦН и УСШН соответственно), доля остальных способов добычи существенно ниже. Определяющим критерием при выборе технологии эксплуатации является дебит скважины, в частности, при дебитах более 50 кубических метров в сутки наибольшим КПД обладает УЭЦН [1-3].
Обеспечение высоких технико-экономических показателей добычи нефти в условиях высокой степени выработки запасов, повышения негативного влияния осложняющих факторов на работу насосного оборудования в значительной степени определяется комплексным решением задач совершенствования технических средств и насосного оборудования, обоснованного выбора технологического режима скважин. Применительно к электроцентробежным насосам (ЭЦН) одним из наиболее распространенных осложнений в работе насосного оборудования является влияние механических примесей, в частности, обусловленное выносом из пласта продуктов механического разрушения пласта при проведении ГРП. Согласно статистическим исследованиям, проведенным на месторождениях Западной Сибири, на долю меха- 
нических примесей приходится до $50 \%$ отказов насосного оборудования. Кроме того, механизированную эксплуатацию скважин значительно осложняют: высокая кривизна стволов скважин, снижение пластового давления в процессе разработки залежей, обуславливающее рост содержания газовой фазы в откачиваемой продукции [4-6].

Для защиты погружного оборудования от механических примесей в составе УЭЦН наибольшее распространение получили скважинные фильтры, наилучшими гидравлическими характеристиками среди которых обладают каркасно-проволочные фильтры. В качестве фильтрующего элемента используется V-образная проволока с треугольным сечением. Благодаря такой геометрии создаются условия для эффективной фильтрации флюида, стимулируется вынос мелких взвешенных частиц и исключается цементация и забивание фильтрующих отверстий. В работе [7] предложена модификация каркаснопроволочного фильтра, отличающегося кривизной проволоки (боковые поверхности треугольного профиля скруглены вовнутрь), причем показано, что усовершенствованная конструкция фильтра обладает лучшими гидравлическими характеристиками в сравнении с фильтром со стандартным треугольным профилем проволоки.

Следует отметить, что на сегодняшний день не существует общепринятой методики расчета технологического режима скважин, оборудованных УЭЦН с механическими фильтрами. Вместе с тем условия эксплуатации погружного оборудования для этих скважин отличаются от условий эксплуатации УЭЦН без влияния осложняющих факторов: за счет влияния гидравлического сопротивления фильтра происходит увеличение давления на приеме насоса и изменяется геометрия линий тока жидкости в области приема ЭЦН. Следовательно, изменяются и особенности движения газовой фазы, а также твердых частиц механических примесей, движущихся в потоке жидкости. Первый фактор оказывает значительное влияние на условия сепарации газа на приеме насоса, что необходимо учитывать при расчете оптимального давления на приеме насоса. Несмотря на значительное количество работ, посвященных сепарации газа, в частности [8, 9], в настоящее время не предложено научно обоснованных и общепринятых методов расчета коэффициента сепарации газа на приеме погружного оборудования с учетом гидравлических характеристик механических фильтров в составе насоса. Исследование влияния механических примесей на работу фильтров (распределения концентрации механических примесей, траектории твердых взвешенных частиц в интервале приема) представляет самостоятельный научный интерес с точки зрения обеспечения оптимальных условий эксплуатации ЭЦН. Особенности многофазного течения газожидкостного потока в трубах в процессе эксплуатации добывающих скважин исследованы в ряде работ [10-13], ряд работ посвящен в том числе различным аспектам влияния твердых частиц механических примесей на особенности формирования полей скорости и концентрации при движении многофазного потока [14-18]. Особенностью данной работы является исследование влияния механических фильтров и их гидравлических характеристик на характер движения газожидкостного потока, содержащего механические примеси, в интервале приема УЭЦН.

\section{Постановка задачи и основные уравнения}

Рассмотрим совместную эксплуатацию ЭЦН с фильтрующей секцией, расположенной между приемной секцией насоса и гидрозащитой (протектором) (рис. 1). Пластовая жидкость движется в кольцевом зазоре между эксплуатационной колонной и насосной установкой и, перетекая через фильтрующую секцию, поступает на прием насоса. Для упрощения численного моделирования полагается, что модульные элементы погружной установки (погружной электродвигатель (ПЭД), гидрозащита, фильтр) примыкают друг к другу непосредственно.

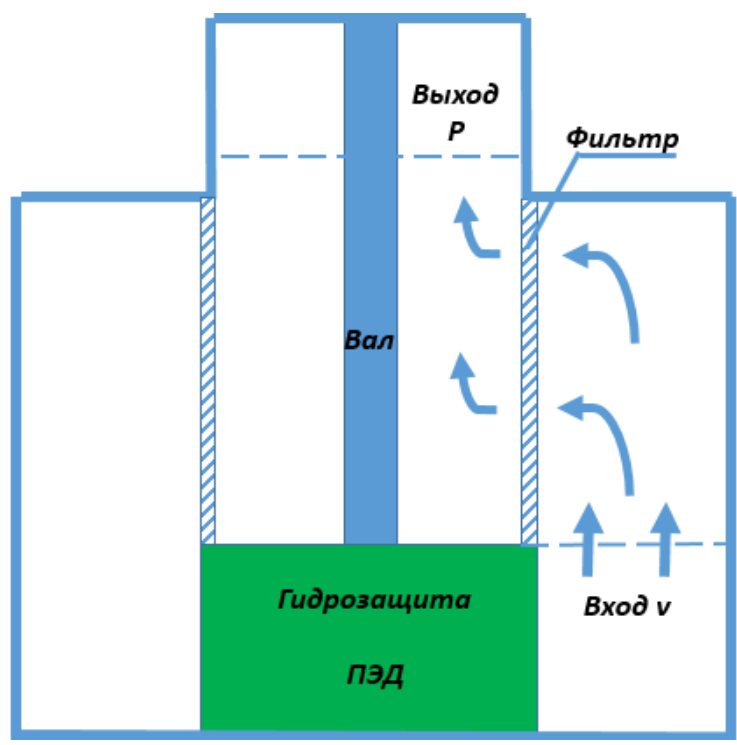

Рис. 1. Расчетная схема течения

Fig. 1. Design diagram of the flow

Расчет полей давления и скорости жидкой фазы базируется на уравнениях Навье-Стокса, записанных в цилиндрической системе координат [19]

$$
\begin{gathered}
\frac{1}{r} \frac{\partial}{\partial r}(r u)+\frac{\partial v}{\partial z}=0 ; \\
\frac{\partial u}{\partial t}+\frac{1}{r} \frac{\partial\left(r \cdot u^{2}\right)}{\partial r}+\frac{\partial(u v)}{\partial z}+\frac{1}{\rho} \frac{\partial p}{\partial r}= \\
=\frac{\mu}{\rho}\left(\frac{1}{r} \frac{\partial}{\partial r}\left(r \frac{\partial u}{\partial r}\right)-\frac{u}{r^{2}}+\frac{\partial^{2} u}{\partial z^{2}}\right)+F_{r} ; \\
\frac{\partial v}{\partial t}+\frac{1}{r} \frac{\partial(r \cdot u v)}{\partial r}+\frac{\partial v^{2}}{\partial z}+\frac{1}{\rho} \frac{\partial p}{\partial z}= \\
=\frac{\mu}{\rho}\left(\frac{1}{r} \frac{\partial}{\partial r}\left(r\left(\frac{\partial v}{\partial r}\right)\right)+\frac{\partial^{2} v}{\partial z^{2}}\right)+F_{z},
\end{gathered}
$$

где $t$ - время; $r$ и $z$ - радиальная и вертикальная координаты соответственно; $\rho$ - плотность жидкости; $u$ и 
$v$ - радиальная и вертикальная компоненты скорости; $p$ - давление жидкости; $\mu$ - коэффициент динамической вязкости водонефтяной смеси; $F_{r}$ и $F_{z}-$ проекции результирующей сил, действующих на жидкость:

$$
\begin{gathered}
F_{r}=-\frac{1}{2} H \frac{\rho u|u|}{\chi^{2}\left(R_{2}-R_{1}\right)}+F_{r s}+F_{r g} ; \\
F_{z}=-\frac{1}{2} H \frac{\rho v|v|}{\chi^{2}\left(R_{2}-R_{1}\right)}-g+F_{z s}+F_{z g} ; \\
\chi=\eta n,
\end{gathered}
$$

где $H$ - дискретная функция, равная единице в области фильтра и нулю в других точках; $R_{1}$ и $R_{2}$ - внутренний и внешний радиус фильтрующего элемента соответственно; $\chi$ - гидравлический параметр фильтра, численно равный произведению скважности $\eta$ (под скважностью фильтра понимают отношение площади отверстий к общей площади боковой поверхности фильтра, выраженное в процентах) и коэффициента расхода $n$; силы $F_{r s}, F_{r g}, F_{z s}, F_{z g}$ действуют на жидкость со стороны газовых пузырьков и твердых частиц, индекс $s$ соответствует фракции механических примесей (sand), индекс $g$ - газовой фазе.

Плотность и вязкость жидкости как водонефтяной смеси определяется как

$$
\begin{aligned}
& \rho=\rho_{o}(1-B)+\rho_{w} B ; \\
& \mu=\mu_{o}(1-B)+\mu_{w} B,
\end{aligned}
$$

где $\rho, \mu$ - плотность и вязкость компонент; индекс $o$ соответствует нефти; индекс $w$ - воде; $B$ - обводненность продукции.

Для расчета компонент скорости пузырьков и частиц механических примесей запишем уравнения баланса сил, действующих на взвешенные в потоке частицы [20]:

$$
\vec{F}_{g r}+\vec{F}_{a}+\vec{F}_{d}+\vec{F}_{p},
$$

где $F_{g r}, F_{a}, F_{d}, F_{p}$ - соответственно сила тяжести, выталкивающая (архимедова) сила, сила сопротивления со стороны жидкости, сила давления, определяемые согласно следующим зависимостям:

$$
\begin{gathered}
\vec{F}_{g r}=\frac{4}{3} \pi r_{s / g}{ }^{3} \rho_{s / g} \vec{g} ; \\
\vec{F}_{a}=\frac{4}{3} \pi r_{s / g}{ }^{3} \rho \vec{g} ; \\
\vec{F}_{d}=\frac{1}{2} C_{d} \rho S_{s / g}\left(\vec{w}-\vec{w}_{s / g}\right)\left|\vec{w}-\vec{w}_{s / g}\right| ; \\
\vec{F}_{p}=\frac{4}{3} \pi r_{s / g}{ }^{3} \frac{\partial p}{\partial x_{i}} \vec{e}_{i},
\end{gathered}
$$

где $r$ - радиус взвешенных частиц (пузырьков газа); $w$ - вектор скорости жидкости; $x_{i}-$ координата (радиальная или вертикальная); $e_{i}-$ единичный вектора соответствующей координаты; $C_{d}-$ коэффициент сопротивления, определяемый числом Рейнольдса; $S$ площадь поперечного сечения пузырьков газа или частиц механических примесей.

Система уравнений (1)-(7) решается численно. В качестве начальных условий принимается равенство нулю скорости всех компонент. На входе модели задается распределение вертикальной скорости потока согласно аналитическому решению уравнения Навье-Стокса, описывающему стационарное движение жидкости в кольцевом пространстве между двумя концентрично расположенными трубами [21]:

$$
u=\frac{r_{2}^{2}-r^{2}}{4 \mu} i-\frac{r_{2}^{2}-R_{2}^{2}}{4 \mu} i \frac{\ln \frac{r}{r_{2}}}{\ln \frac{R_{2}}{r_{2}}},
$$

где $r_{2}$ - внутренний радиус обсадной колонны; $i-$ вертикальный градиент давления, определяемый по заданному объемному расходу жидкости $Q$ через кольцевое сечение.

На поверхности обсадной колонны $\left(r=r_{2}\right)$, ПЭД и гидрозащиты $\left(r=R_{2}\right)$ принимаются условия прилипания, на поверхности вала $\left(r=r_{1}\right)$ и на входе в затрубное пространство НКТ - условия непротекания.

\section{Анализ результатов расчетов}

Моделирование течения газожидкостного потока в интервале приема насоса выполняется при следующих значениях геометрических и технологических параметров скважины и УЭЦН: радиус вала $r_{1}=0,01$ м, радиус обсадной колонны $r_{2}=0,07 \mathrm{M}$, внутренний и внешний радиусы фильтра $R_{1}=0,045, R_{2}=0,050$ м, дебит жидкости $Q=80 \mathrm{~m}^{3} /$ сут, обводненность продукции $B=0,5$, плотность нефти $\rho_{o}=800 \mathrm{\kappa} / \mathrm{m}^{3}$, плотность воды $\rho_{w}=1000 \mathrm{\kappa г} / \mathrm{m}^{3}$, вязкость нефти $\mu_{o}=0,01$ Па с , вязкость воды $\mu_{w}=0,001$ Па с, радиус пузырьков газа $r_{g}=100$ мкм, радиус частиц механических примесей $r_{s}=150$ мкм, ускорение свободного падения $g=9,8 \mathrm{~m} / \mathrm{c}^{2}$.

Исследуется многофазное течение потока для трех вариантов оборудования приемного модуля:

1) без механического фильтра;

2) с каркасно-проволочным фильтром со стандартным треугольным профилем проволоки (параметры фильтра: гидравлический параметр $\chi=0,06$, коэффициент расхода $\eta=0,2$, скважность фильтра $s=0,3)$;

3) с каркасно-проволочным фильтром с усовершенствованным треугольным профилем проволоки (параметры фильтра: гидравлический параметр $\chi=0,24$, коэффициент расхода $\eta=0,8$, что в 4 раза выше, чем для стандартного фильтра при той же скважности фильтра $s=0,3)$.

На графиках (рис. 2-4) показано распределение радиальной, вертикальной скорости при обтекании жидкости погружной части УЭЦН.

Характер течения жидкой фазы показывает, что при движении в интервале фильтра поток разделяется на два части: одна устремляется непосредственно в приемные отверстия, а вторая движется вдоль приемного модуля параллельно его оси к верхнему интервалу. Наличие фильтра в составе насоса значительно изменяет геометрию течения жидкости на приеме. Для насоса с фильтром за счет дополнительного гидравлического сопротивления, создаваемого фильтром, интервал фильтра работает более равномерно (рис. 3,4$)$, причем по мере снижения гидравлического параметра $\chi$ достигается более равномерный профиль радиального притока жидкости к фильтру (рис. 4). 

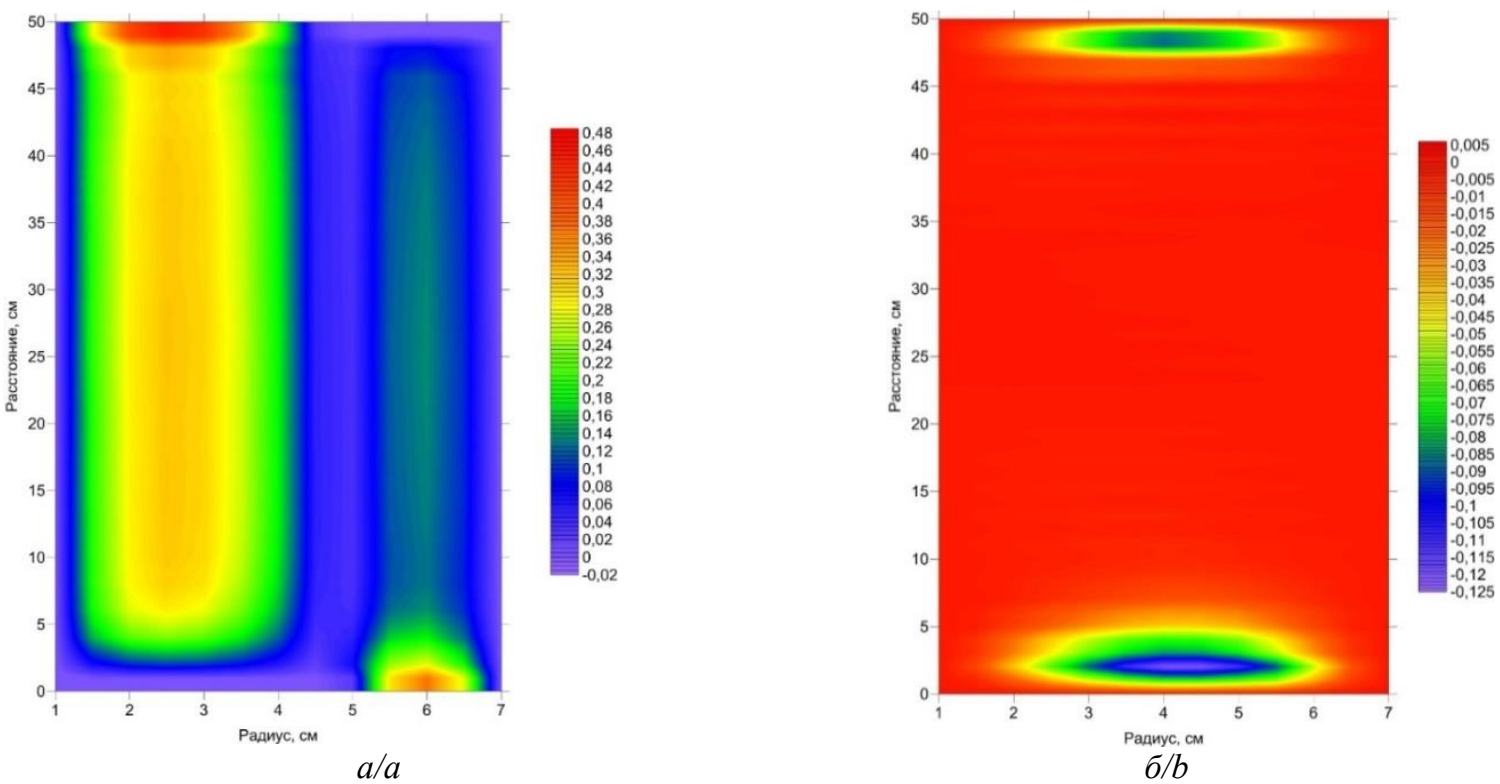

Pис. 2. Распределение вертикальной (а) и радиальной (б) компонент скорости жидкости в интервале приема насоса (без фильтра)

Fig. 2. Distribution of the vertical (a) and radial (b) components of the fluid velocity in the pump intake interval (without screen)
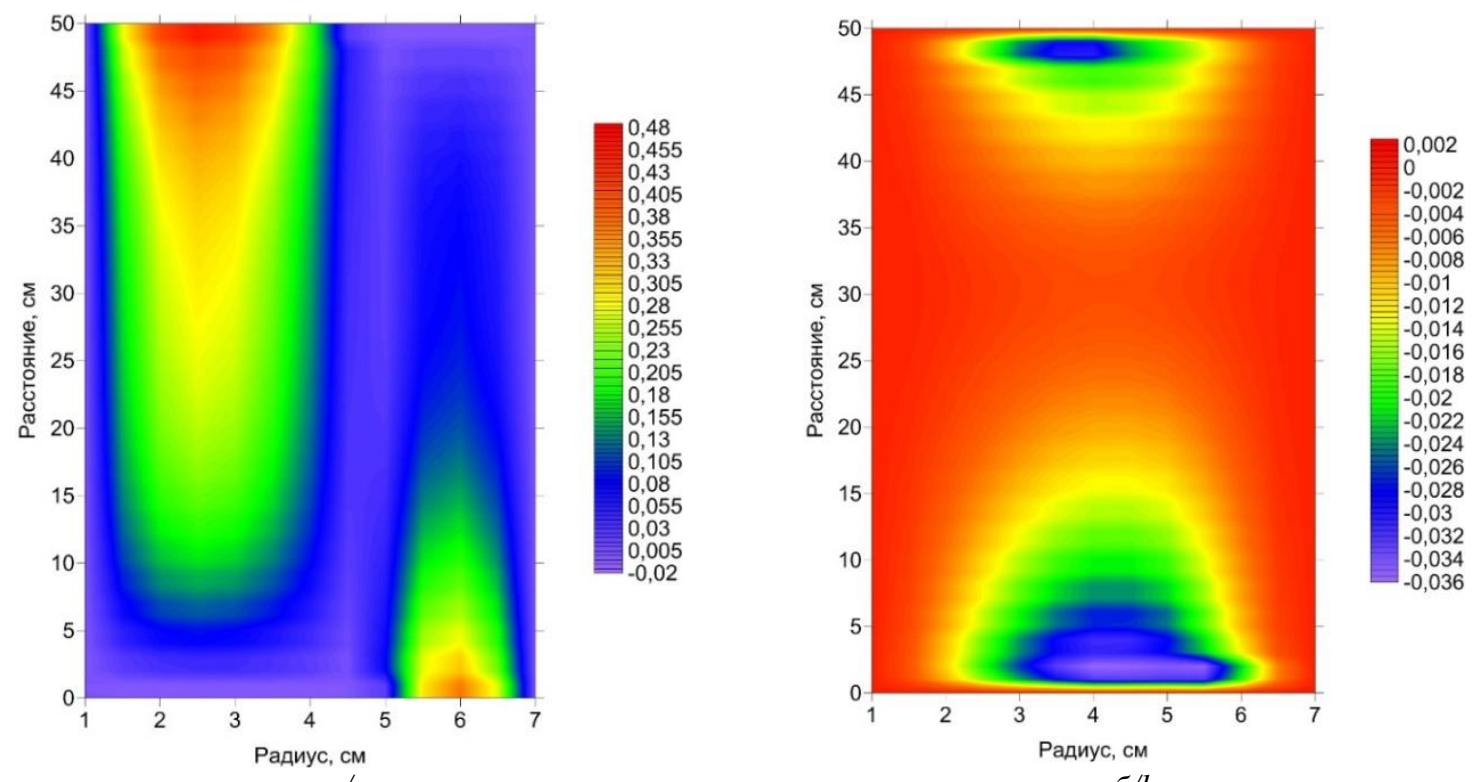

$a / a$

$\sigma / b$

Pис. 3. Распределение вертикальной (а) и радиальной (б) компонент скорости жидкости в интервале приема насоса (с каркасно-проволочным фильтром, гидравлический параметр фильтра $\chi=0,24)$

Fig. 3. Distribution of the vertical (a) and radial (b) components of the fluid velocity in the pump intake interval (with wirewound screen, hydraulic filter parameter $\chi=0,24)$

Расчет траектории частиц жидкости (рис. 5), поступающих к интервалу приема из различных кольцевых сечений потока в обсадной колонне, показывает, что частицы жидкости, изначально движущиеся вблизи приемных отверстий, поступают в насос через нижние отверстия, частицы, движущиеся вблизи стенки обсадной колонны, - через верхние. Кроме того, из графиков следует, что изменение гидравлических характеристик фильтра обуславливает видимое изменение геометрии линий тока в интервале приема насоса.
Траектория пузырьков газа, движущихся в потоке жидкости, определяется соотношением силы сопротивления со стороны жидкости, «увлекающей» газ в направлении приемного модуля, и силы Архимеда, обуславливающей подъем пузырьков газа и его сепарацию в затрубное пространство. Из графиков (рис. 6) следует, что при моделируемых условиях пузырьки газа, движущиеся вблизи приемных отверстий (красная и зеленая штриховые линии на рис. 6), увлекаются жидкостью к приемному модулю. Напротив, пузырьки газа, находящиеся вблизи обсадной колонны, 
всплывают в потоке жидкости, сепарируясь в затрубное пространство, и не поступают на прием насоса (синяя линия на рис 6). Расчетный коэффициент сепарации газа составил 0,38 для фильтра с гидравлическим параметром $\chi=0,24$ и соответственно 0,32 для

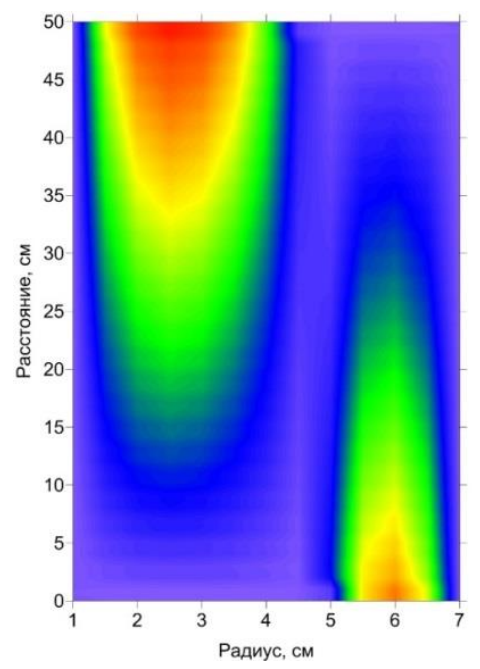

$a / a$ фильтра с гидравлическим параметром $\chi=0,06$. Следует отметить, что расчетная величина коэффициента сепарации отличается от оценки по известной формуле Мищенко (0.4), получившей широкое распространение в инженерных расчетах при нефтедобыче $[1,2]$.

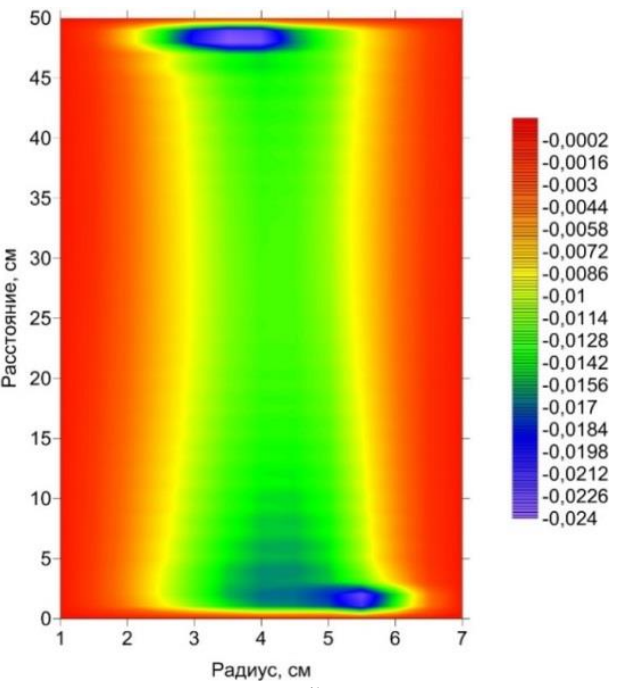

$\sigma / b$

Рис. 4. Распределение вертикальной (а) и радиальной (б) компонент скорости жидкости в интервале приема насоса (с каркасно-проволочным фильтром, гидравлический параметр фильтра $\chi=0,06$ )

Fig. 4. Distribution of the vertical $(a)$ and radial $(b)$ components of the fluid velocity in the pump intake interval (with wirewound screen, filter hydraulic parameter $\chi=0,06)$

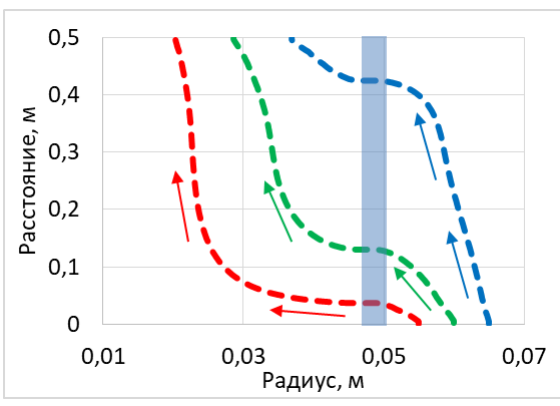

$a / a$

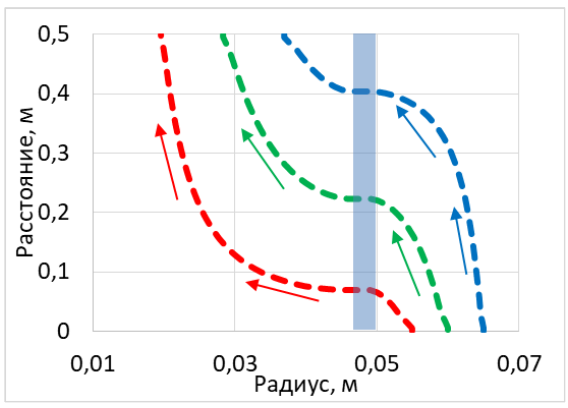

$\sigma / b$

Рис. 5. Траектория движения частии жидкости в интервале приеме (гидравлический параметр фильтра (a) $\chi=0,24$, (б) $\chi=0,06)$, закраска - область фильтра

Fig. 5. Motion path of liquid particles in the intake interval (hydraulic filter parameter (a) $\chi=0,24$, (b) $\chi=0,06$ ), shading filter area

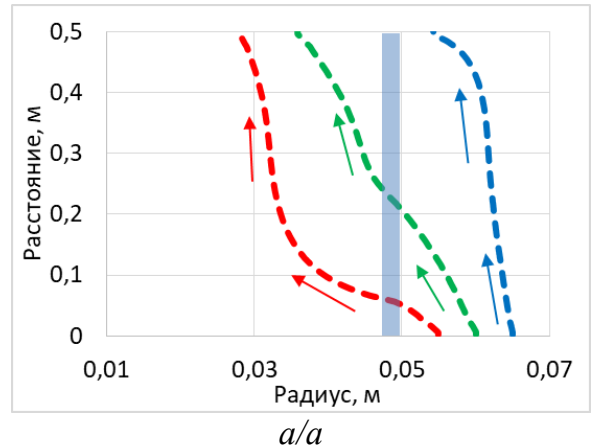

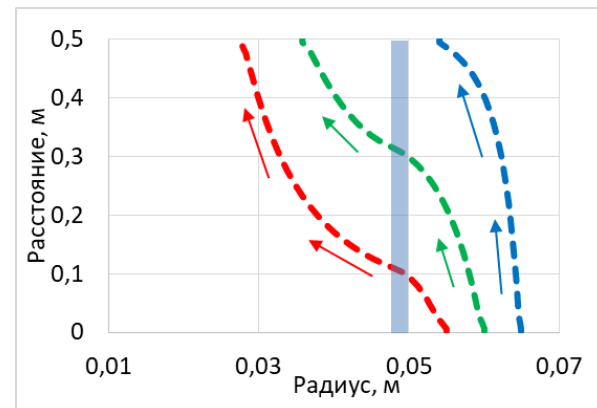

$\sigma / b$

Рис. 6. Сравнение траектории движения пузырьков газа в интервале приема (гидравлический параметр фильтра (a) $\chi=0,24$, (б) $\chi=0,06$ )

Fig. 6. Comparison of the motion path of gas bubbles in the intake interval (hydraulic filter parameter (a) $\chi=0,24$, (b) $\chi=0,06$ ) 
Характер движения твердых взвешенных частиц в газожидкостном потоке определяется совместным влиянием силы тяжести, архимедовой силы и силы сопротивления жидкости движению частиц. Величина силы тяжести превосходит архимедову, поэтому результирующая этих двух сил направлена вертикально вниз, в связи с чем скорость подъема механических примесей (МП) снижается относительно скорости жидкости. В результате частицы МП в обсадной колонне концентрируются в основном у нижних отверстий фильтра (рис. 7). Поскольку область внутри фильтра у его нижних отверстий характеризуется

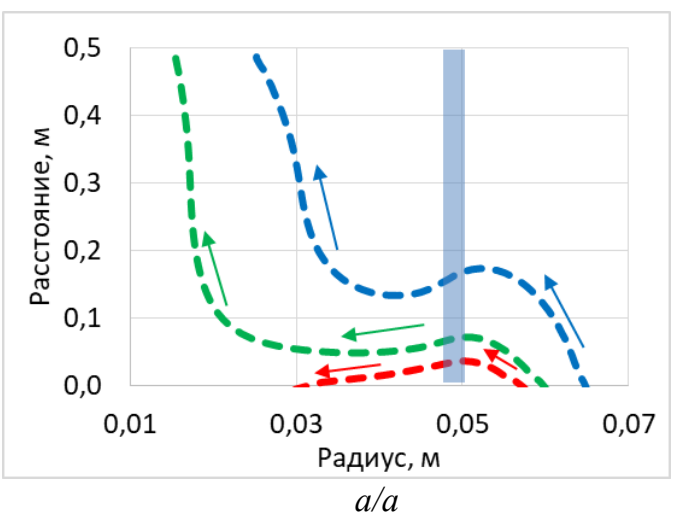

низкой скоростью потока, недостаточной для подъема твердых взвешенных частиц, механические примеси, попадающие в эту область, оседают в нижнем конце фильтра и накапливаются (красная линия на рис. 7), что приводит к образованию «застойной зоны», затрудняющей движение жидкости в этом интервале. Кроме того, из рис. 7, б видно, что при малом гидравлическом параметре $(\chi=0,06)$ вследствие низкой скорости потока жидкости внутри фильтра в его нижней части частицы механических примесей накапливаются более интенсивно (как следует из траектории зеленой линии на рис. 7, $a$, б).

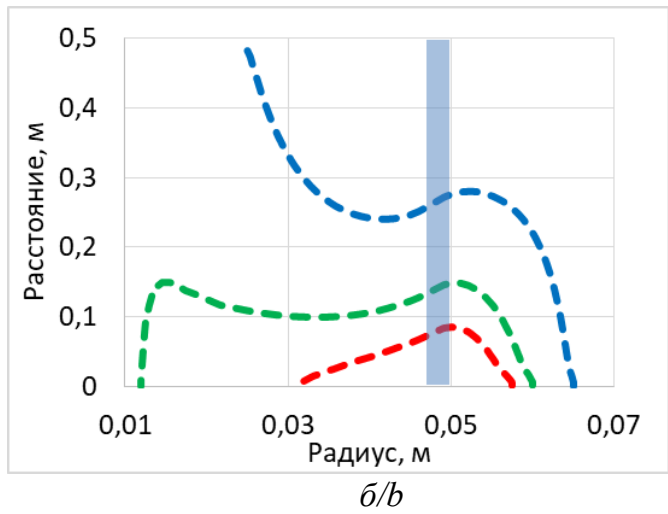

Рис. 7. Сравнение траектории движения взвешенных частии механических примесей (гидравлический параметр фильтра (а) $\chi=0,24$, (б) $\chi=0,06$ )

Fig. 7. Comparison of the motion path of suspended particles of mechanical impurities (hydraulic filter parameter (a) $\chi=0,24$, (b) $\chi=0,06$ )

Исследование влияния дебита по жидкости (объемного расхода восходящего потока) на размер частиц механических примесей, которые будут оседать и накапливаться внутри рассмотренных конструктивных вариантов фильтров, показано на рис. 8 .

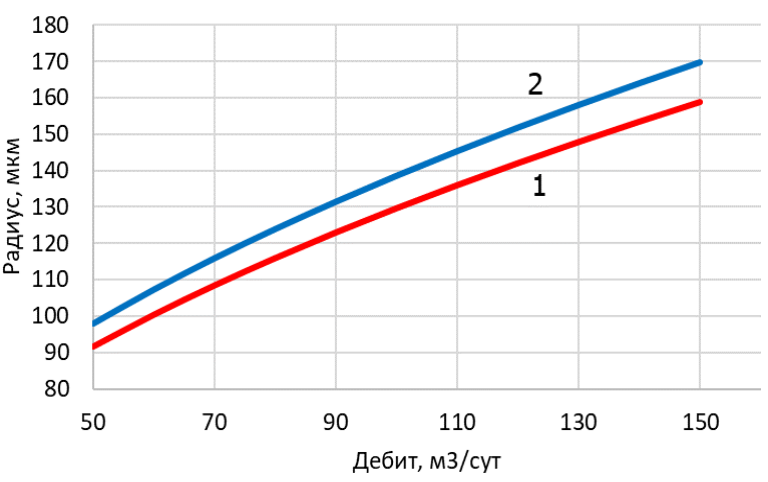

Pис. 8. Минимальный радиус оседаюших в фильтре частии при различном дебите восходящего потока $(1-\chi=0,06,2-\chi=0,24)$

Fig. 8. Minimum radius of particles settling in the filter at different upstream flow rate $(1-\chi=0,06,2-\chi=0,24)$

Видно, что размер частиц, поднимаемых восходящим потоком, закономерно возрастает с увеличением дебита жидкости. При дебите $50 \mathrm{~m}^{3} /$ сут в фильтре будут оседать частицы диаметром 180-200 мкм и более, при дебите $100 \mathrm{~m}^{3} /$ сут - диаметром более 260-270 мкм. Для исключения накопления механиче- ских примесей размер фильтрующих отверстий не должен превышать минимальный диаметр частиц, оседающих в фильтре при данном дебите восходящего потока. Например, при дебите $50 \mathrm{~m}^{3} /$ сут размер фильтрующих отверстий не должен превышать 180 мкм. Для каркасно-проволочного фильтра с диаметром проволоки 5 мм и межвитковым расстоянием 180 мкм это будет соответствовать фильтру скважностью $s=0,036$. Таким образом, особенности движения механических примесей в интервале приема насоса с фильтром необходимо учитывать для обоснованного выбора его конструктивных параметров. Из рис. 8 видно также, что увеличение гидравлического параметра фильтра и улучшение его гидравлических характеристик приводит к росту размера поднимаемых в фильтре частиц (на величину порядка 10 \%).

При движении жидкости через отверстия фильтра возникают гидравлические потери, причем перепад давления на фильтрующем элементе определяется в общем конструктивными особенностями фильтра, размером фильтрующих отверстий, дебитом жидкости и реологическими свойствами откачиваемого флюида. Для рассмотренных вариантов фильтров каркасно-проволочного усовершенствованного (с коэффициентом расхода $\eta=0,8)$ и стандартного $(\eta=0,2)-$ исследовано влияние скважности фильтра $s$ на величину потерь давления при прохождении жидкости через фильтр (рис. 9). В расчетах для простоты коэффициент расхода принят постоянным и не зависящим от скважности. 


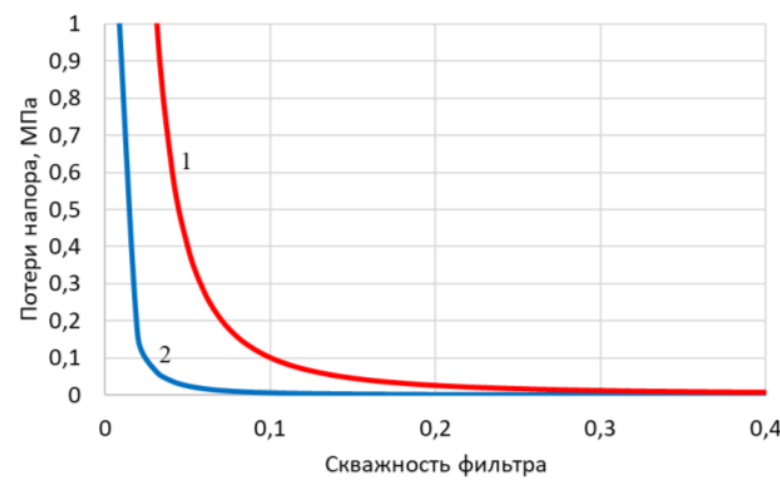

Puc. 9. Зависимость потерь давления от скважности для фильтров различных модификаций (1- $\eta=0,2$, $2-\eta=0,8)$

Fig. 9. Dependence of filter pressure losses on the borehole for various modifications $(1-\eta=0,2,2-\eta=0,8)$

С уменьшением размера фильтрующих отверстий (и соответственно скважности) перепад давления на фильтре возрастает, причем на величину потерь давления существенное влияние оказывают его конструкционные особенности, определяемые коэффициентом расхода $\eta$. Например, для фильтра с коэффициентом расхода $\eta=0,8$ при скважности $0,05(\chi=0,04)$ потери давления на фильтре составляют $0,025 \mathrm{MПа,}$ при более низком коэффициенте расхода $(\eta=0,2$, $\chi=0,01)$ потери давления возрастают до 0,4 МПа. Увеличение потерь напора в фильтре, как известно, ведет к росту забойного давления, снижению депрессии на пласт и притока пластовой жидкости, следовательно, снижению напорной характеристики насоса. Таким образом, совершенствование конструкции скважинных фильтров и улучшение их гидравлических характеристик - важные задачи с точки зрения повышения эффективности насосной эксплуатации скважин.

\section{Выводы}

1. Разработана математическая модель течения газожидкостного потока, содержащего взвешенные частицы механических примесей, в интервале приема электроцентробежного насоса, оборудованного каркасно-проволочным фильтром. Качественно исследовано влияние гидравлических параметров фильтра на особенности течения много-

\section{СПИСОК ЛИТЕРАТУРЫ}

1. Takacs G. Electrical submersible pumps manual. - Houston, Texas: Gulf Professional Publishing, 2017. - 578 p.

2. Методы защиты насосного оборудования для добычи нефти от механических примесей / С.В. Смольников, А.С. Топольников, К.Р. Уразаков, Р.Н. Бахтизин. - Уфа: Нефтегазовое дело, 2010. $-41 \mathrm{c}$.

3. Учет условий эксплуатации при проектировании периодических режимов работы скважин, оборудованных УЭЦН / В.Н. Ивановский, А.А. Сабиров, С.Б. Якимов, А.А. Клусов // Оборудование и технологии для нефтегазового комплекса. 2013. - № 6. - C. 33-39.

4. Бахтизин Р.Н., Нургалиев Р.З., Уразаков К.Р. Эксплуатация насосных скважин, осложненных механическими примесями. - Уфа: Изд-во УГНТУ, 2016. - 91 с.

5. Dynamic model of a Rod Pump Installation for inclined wells R.N. Bakhtizin, K.R. Urazakov, S.F. Ismagilov, A.S. Topol'nikov, F.F. Davletshin // SOCAR Proceedings. - 2017. - № 4. - P. 74-82 компонентного потока в интервале приема насоса. Показано, что по мере снижения гидравлического параметра достигается более равномерный профиль радиального притока жидкости в интервале фильтра, причем потери напора при прохождении жидкости через фильтр не превышают 0,07 МПа.

2. Показано, что условия сепарации газа в интервале приема, а также распределение концентрации механических примесей изменяются при варьировании гидравлических характеристик фильтра. Расчетный коэффициент сепарации газа при варьировании гидравлического параметра фильтра в интервале 0,06-0,024 изменился в пределах 0,32-0,38, причем расчетная величина коэффициента сепарации отличается от оценки по известной формуле Мищенко $(0,40)$, получившей широкое распространение в инженерных расчетах при нефтедобыче.

3. Установлено, что за счет значительного влияния силы тяжести частицы механических примесей в обсадной колонне концентрируются в основном у нижних отверстий фильтра. Показано, что внутри фильтра у его нижних отверстий может создаваться застойная зона, характеризующаяся низкой скоростью потока, недостаточной для подъема твердых взвешенных частиц, в результате чего механические примеси, попадающие в эту область, оседают в нижнем конце фильтра. Установлено, что при увеличении дебита восходящего потока с 50 до $100 \mathrm{~m}^{3} /$ сут минимальный диаметр оседающих в фильтре частиц возрастает со 180-200 до 260-270 мкм, в зависимости от гидравлического параметра фильтра.

4. Показано, что потери давления на фильтрующем элементе возрастают по мере снижения скважности и коэффициента расхода фильтра, причем при снижении гидравлического параметра фильтра с 0,04 до 0,01 потери давления возрастают с 0,025 до 0,4 МПа. Таким образом, совершенствование конструкции скважинных фильтров является важной задачей с точки зрения повышения эффективности механизированной эксплуатации скважин, в том числе электроцентробежными установками (УЭЦН).

6. Особенности работы скважинных сепараторов механических примесей в составе скважинных штанговых насосных установок / А.В. Булат, А.В. Деговцов, В.Н. Ивановский, Д.О. Корольков, А.А. Сабиров // Территория «НЕФТЕГАЗ». - 2018. № 7-8. - C. 42-52.

7. Исследование характеристики каркасно-проволочного фильтра численным гидродинамическим моделированием / Р.3. Нургалиев, Р.Н. Бахтизин, К.Р. Уразаков, А.Г. Губайдуллин // Нефтяное хозяйство. - 2017. - № 10. - С. 113-115.

8. Волков М.Г., Михайлов В.Г., Петров П.В. Исследование влияния структуры газожидкостной смеси на эффективность процесса сепарации газа в центробежном газосепараторе // Вестник УГАТУ. Машиностроение. - 2012. - Т. 16. № 5 (50). - С. 93-99.

9. Волков М.Г. Методика расчёта коэффициента естественной сепарации в процессе освоения нефтедобывающей скважины // Нефтегазовое дело. - 2016. - Т. 14. - № 4. - С. 45-49. 
10. Hasan A.R., Kabir C.S. Two-phase flow in vertical and inclined annuli // International Journal of Multiphase Flow. - 1992. V. 18. - P. 279-293.

11. Brill J.P., Mukherjee H. Multiphase flow in wells. - Richardson, Texas: Society of petroleum engineers, 1999. $-384 \mathrm{p}$.

12. Drift-flux parameters for threephase steady-state flow in wellbores / H. Shi, J. Holmes, L. Diaz, L.J. Durlofsky, K. Aziz // Society of petroleum engineers journal. - 2005. - V. 10 (2). - P. 130-137.

13. Смирнова Е.В., Марчевский И.К., Бондарчук В.О. Моделирование осесимметричных течений вязкой несжимаемой жидкости методом конечных элементов с частицами PFEM-2 в программном комплексе Kratos с открытым кодом // Труды Института системного программирования РАН. - 2018. - Т. 30. Вып. 2. - С. 263-284.

14. Моделирование течения запыленного воздуха в респираторном тракте / П.В. Трусов, Н.В. Зайцева, М.Ю. Цинкер, А.В. Бабушкина // Российский журнал биомеханики. - 2018. № 3. - C. 301-314.

15. Yang Y., Peng H., Wen Ch. Sand transport and deposition behaviour in subsea pipelines for flow assurance // Energies. 2019. - V. 21. - № 12. - P. 1-12.

16. On the numerical simulation of sand transport in liquid and multiphase pipelines / M. Leporinia, A. Terenzi, B. Marchetti, F.
Corvaro, F. Polonara // Journal of Petroleum Science and Engineering. - 2019. - V. 175. - P. 519-535.

17. Dabirian R., Mohan R.S., Shoham O. On the numerical simulation of sand transport in liquid and multiphase pipelines // Journal of Petroleum Science and Engineering. - 2017. - V. 156. - P. 721-731.

18. Viscosity eff ects on sand flow regimes and transport velocity in horizontal pipelines / E. Zorgani, H. Al-Awadi, W. Yan, S. Al-Lababid, H. Yeung, C.P. Fairhurst // Experimental Thermal and Fluid Science. - 2018. - V. 92. - P. 89-96.

19. Топольников А.С., Уразаков К.Р., Казаков Д.П. Численное моделирование обтекания погружной части установок электроцентробежных насосов с фильтром // Нефтегазовое дело. - 2009. - Т. 7. - № 2. - С. 89-95.

20. Лугуманов Т.Т., Кулешов В.С. К моделированию динамики системы флюид-дисперсная среда // Труды Института механики имени Р.Р. Мавлютова Уфимского Научного центра Российской Академии Наук. - 2014. - № 10. - С. 66-72.

21. Бахтизин Р.Н., Давлетов М.Ш., Хакимов Т.А. Методика расчета нагрузок на колонну насосных штанг в насосной установке с выносным утяжелителем // Нефтегазовое дело. 2013. - T. 11. - № 2. - C. 59-63.

Поступила 22.09.2021 г.

\section{Информация об авторах}

Уразаков К.Р., доктор технических наук, профессор, профессор кафедры машин и оборудования нефтегазовых промыслов, Уфимский государственный нефтяной технической университет.

Тугунов П.М., ассистент кафедры машин и оборудования нефтегазовых промыслов, Уфимский государственный нефтяной технической университет.

Алиметов Ш.А., инженер-технолог, «Когалымнефтегаз», Общество с ограниченной ответственностью «ЛУКОЙЛ - Западная Сибирь». 
UDC 622.279

\title{
SIMULATION OF GAS-LIQUID FLOW AT THE INTAKE OF ELECTRIC CENTRIFUGAL PUMPING UNITS WITH WIRE-FRAME FILTER
}

\author{
Kamil R. Urazakov ${ }^{1}$ \\ urazakk@mail.ru
}

Pavel M. Tugunov 1 , PavelTugunov@gmail.com

\author{
Shamil A. Alimetov², \\ shamil_alimetov@mail.ru \\ 1 Ufa State Petroleum Technological University, \\ 1, Kosmonavtov street, Ufa, 450064, Russia. \\ 2 Kogalymneftegaz, Limited Liability Corporation «LUKOIL - Western Siberia», \\ 20, Pribaltiyskaya street, Kogalym, Russia.
}

\begin{abstract}
Relevance. Up to date, one of the most unfavorable factors complicating artificial oil lift is the interaction of mechanical impurities carried by production fluid with downhole equipment assemblies. According to statistical studies, mechanical impurities are the determining cause of malfunctions in the electric-centrifugal pump units operation.

Object: wire-wound screen with the best hydraulics compared to other types of mechanical filters. Despite its widespread use, today there is no generally accepted method for calculating the operating practices of wells equipped with electric-centrifugal pumps with mechanical filters. At the same time, paper shows that mechanical filters, due to hydraulic resistance, have a significant effect on the geometry of the fluid flow lines in the pump suction area, therefore, the gas phase and the fraction of mechanical impurities moving in the flow.

Purpose: investigation of the influence of the hydraulic characteristics of mechanical filters on the features of the flow of pumped-out products in the electric-centrifugal pump interval; development of a mathematical model of multicomponent gas-liquid flow containing mechanical impurities in the interval of receiving electric-centrifugal pumping unit equipped with a wire-wound screen.

Results. It was shown that for a pump with a wire-wound screen due to the hydraulic resistance created by it, the intake interval works more evenly, and as the hydraulic parameter decreases, a more uniform profile of the radial fluid inflow in the pump suction area is achieved. The features of the flow of gas bubbles and suspended particles of mechanical impurities moving in the flow of liquid in the area of the pump suction area are investigated. It is shown that the conditions for gas separation at the intake, as well as the distribution of the concentration of mechanical impurities, change when the hydraulic characteristics of the screen in the pump are varied, which must be taken into account when designing the well operation parameters. The influence of the filter hydraulic characteristics on the value of pressure losses during liquid flow through the filter element is investigated.
\end{abstract}

\section{Key words:}

Installation of an electric center pump, wire-frame filter, flow simulation, flow rate, mechanical impurities, gas bubbles, Navier-Stokes equations.

\section{REFERENCES}

1. Takacs G. Electrical submersible pumps manual. Houston, Texas, Gulf Professional Publ., 2017. 578 p.

2. Smolnikov S.V., Topolnikov A.S., Urazakov K.R., Bakhtizin R.N Metody zashchity nasosnogo oborudovaniya dlya dobychi nefti ot mekhanicheskikh primesey [Methods for protecting pumping equipment for oil production from mechanical impurities.]. Ufa, Neftegazovoe delo Publ., 2010. 41 p.

3. Ivanovskii V.N., Sabirov A.A., Yakimov S.B., Klusov A.A. Taking into account the operating conditions in the design of periodic operating modes of wells equipped with ESP. Equipment and technologies for the oil and gas complex, 2013, no. 6, pp. 3339. In Rus.

4. Bakhtizin R.N., Nurgaliev R.Z., Urazakov K.R. Ekspluatatsiya nasosnykh skvazhin, oslozhnennykh mekhanicheskimi primesyami [Operation of pumping wells complicated by mechanical impurities]. Ufa, UGNTU Publ., 2016. 91 p.

5. Bakhtizin R.N., Urazakov K.R., Ismagilov S.F., Topol'nikov A.S. Davletshin F.F. Dynamic model of a Rod Pump Installation for inclined wells. Socar Proceedings, 2017, no. 4, pp. 74-82.

6. Bulat A.V., Degovtsov A.V., Ivanovskiy V.N., Korolkov D.O., Sabirov A.A. Features of the work of well separators mechanical impurities in the composition of sucker-rod pumping unit. Oil and Gas Territory, 2018, no. 7-8, pp. 42-52. In Rus.

7. Nurgaliev R.Z., Bakhtizin R.N., Urazakov K.R., Gubaidullin A.G. Investigation of the characteristics of wire-frame filter by numerical hydrodynamic modeling. Oil Industry, 2017, no. 6, pp. 113-115. In Rus.

8. Volkov M.G., Mikhailov V.G., Petrov P.V. Investigation of the influence of the structure of a gas-liquid mixture on the efficiency of the gas separation process in a centrifugal gas separator. Vestnik UGATU. Mechanical engineering, 2012, vol. 16, no. 5 (50), pp. 93-99. In Rus.

9. Volkov M.G. Methodology for calculating the natural separation coefficient in the process of developing an oil producing well. Petroleum Engineering, 2016, vol. 14, no. 4, pp. 45-49. In Rus.

10. Hasan A.R., Kabir C.S. Two-phase flow in vertical and inclined annuli. International Journal of Multiphase Flow, 1992, vol. 18, pp. 279-293.

11. Brill J.P., Mukherjee H. Multiphase flow in wells. Richardson, Texas, Society of petroleum engineers, 1999. $384 \mathrm{p}$.

12. Shi H., Holmes J., Diaz L., Durlofsky L.J., Aziz K. Drift-flux parameters for threephase steady-state flow in wellbores. Society of petroleum engineers Journal, 2005, vol. 10, no. 2, pp. 130-137.

13. Smirnova E.V., Marchevskii I.K., Bondarchuk V.O. Modeling of axisymmetric flows of a viscous incompressible fluid by the finite element method with PFEM-2 particles in the open-source Kratos software package. Proceedings of the Institute of system programming of the Russian Academy of Sciences, 2018, vol. 30, no. 2, pp. 263-284. In Rus.

14. Trusov P.V., Zaitseva N.V., Tsinker M.Yu., Babushkina A.V. Modeling the flow of dusty air in the respiratory tract. Russian journal of biomechanics, 2018, no. 3, pp. 301-314. In Rus. 
15. Yang Y., Peng H., Wen Ch. Sand transport and deposition behaviour in subsea pipelines for flow assurance. Energies, 2019, vol. 21 , no. 12 , pp. $1-12$.

16. Leporinia M., Terenzi A., Marchetti B., Corvaro F., Polonara F. On the numerical simulation of sand transport in liquid and multiphase pipelines. Journal of Petroleum Science and Engineering, 2019, vol. 175, pp. 519-535.

17. Dabirian R., Mohan R.S., Shoham O. On the numerical simulation of sand transport in liquid and multiphase pipelines. Journal of Petroleum Science and Engineering, 2017, vol. 156, pp. 721-731.

18. Zorgani E., Al-Awadi H., Yan, W., Al-Lababid S., Yeung, H. Fairhurst C.P. Viscosity effects on sand flow regimes and transport velocity in horizontal pipelines. Experimental Thermal and Fluid Science, 2018, vol. 92, pp. 89-96.
19. Topolnikov A.S., Urazakov K.R., Kazakov D.P. Numerical modeling of the flow around the submersible part of the installations of electric centrifugal pumps with a filter. Petroleum Engineering, 2009, vol. 7, no. 2, pp. 89-95. In Rus.

20. Lugumanov T.T., Kuleshov V.S. On modeling the dynamics of the fluid-dispersed medium system. Proceedings of the R.R. Mavlyutov Institute of mechanics of the Ufa Scientific center of the Russian Academy of Sciences, 2014, no. 10, pp. 66-72. In Rus.

21. Bakhtizin R.N., Davletov M.Sh., Khakimov T.A. Method of calculating loads on a column of pumping rods in a pumping unit with a remote weighting device. Oil and Gas business, 2013, vol. 11, no. 2, pp. 59-63. In Rus.

Received: 22 September 2021.

\section{Information about the authors}

Kamil R. Urazakov, Dr. Sc., professor, Ufa State Petroleum Technological University.

Pavel M. Tugunov, assistant, Ufa State Petroleum Technical University.

Shamil A. Alimetov, process engineer, Kogalymneftegaz, Limited Liability Corporation «LUKOIL - Western Siberia». 